\title{
Special Project Images
}

\begin{tabular}{llll}
\hline $\begin{array}{l}\text { Area } \\
\text { Name }\end{array}$ & Image & Image & $\begin{array}{l}\text { Identification } \\
\text { Number }\end{array}$ \\
\hline
\end{tabular}

\section{States}

Alaska - mosaic w/map scale, cities

AVHRR

$01 / 91$

E-1925-66CT

Florida - full State mosaic

Florida - southern mosaic including Orlando

Landsat MSS

$04 / 75-05 / 85$

Landsat TM

$03 / 92$

Florida - southern mosaic w/1:250,000-scale map

Landsat TM

$03 / 92-04 / 93$

E-1721-99CT

Hawaiian Islands - oblique of all islands

Shuttle

$05 / 85$

South Dakota - mosaic

Landsat MSS

$05 / 78$

E-1930-99CT

South Dakota - mosaic with map grid

Landsat TM

1986-1993

E-1739-99CT

E-439-58CT

Cities

Athens, Greece

Landsat MSS

$08 / 72$

E-1994-47CT

\section{Beijing, China}

Baghdad, Iraq

Cairo, Egypt

Landsat MSS

$05 / 91$

Landsat TM/HRV

$01 / 91$

Chicago, IL

Landsat TM

$11 / 86$

Chicago, IL

Landsat MSS

$10 / 75$

Damascus, Syria

NASA Skylab

$01 / 74$

Denver, CO

Kiev, USSR

Las Vegas, NV

Landsat MSS

$12 / 78$

Landsat MSS $\quad 10 / 76$

Landsat TM $\quad 04 / 86$

Landsat TM $\quad 04 / 83$

Los Angeles, CA

Mexico City, Mexico

Landsat TM

$11 / 87$

Landsat TM $\quad 01 / 85$

New Orleans, LA

Landsat MSS

$04 / 76$

Aircraft

New Orleans, LA

Landsat MSS

N/A

\begin{tabular}{ll} 
NASA Aircraft & $03 / 84$ \\
\hline
\end{tabular}

New York, NY - oblique

NASA Skylab

Phoenix, AZ

Pierre, SD

Rome, Italy

Landsat MSS

$01 / 74$

Landsat MSS $\quad 06 / 75$

San Diego Bay, CA

Landsat TM

NASA Airctaft

San Francisco, CA - oblique

San Francisco Bay, CA - mosaic

Sioux Falls, SD - mosaic*

Stockholm, Sweden

Tokyo, Japan

Washington, DC - low oblique

Washington, DC - vertical

Washington, DC - high oblique

NASA Aircraft

$04 / 72$

Landsat TM 07/85

Aircraft $\quad 04 / 90$

Landsat MSS $\quad 05 / 75$

Landsat MSS $11 / 72$

Aircraft $\quad 02 / 84$

Aircraft $\quad 02 / 84$

Aircraft $\quad 07 / 83$

E-567-99CT

E-1943-99CT

E-1846-99CT

E-1597-99CT

E-1876-99CT

E-1370-99CT

E-1346-99CT

E-1829-99CT

E-1532-55CT

E-1469-46CT

E-1702-99CT

E-1507-99CT

E-1832-99CT

E-149-810CT

E-1478-99CT

E-1704-99CT

E-1372-99CT

E-1833-99CT

E-1834-99CT

E-1807-99CT

E-1703-99CT

E-1814-99CT

E-772-99BN

E-1835-99CT

E-1836-99CT

E-1567-99CT

E-1568-99CT

E-1569-99CT

\section{Special Topics-Space}

Africa/Nile River - with Hubble satellite in Discovery

Cargo Bay

Shuttle over the Andes

Andes Mts., S. America

Astronaut W/Flag on Moon

Columbia Landing

Earth Rise From Moon

Full Moon

Saturn/Jupiter - mosaic of planets

Space Shuttle Columbia III - launch with reflection in water
Apollo

Shuttle

Apollo

Apollo

Shuttle
$04 / 90$

$04 / 90$

$12 / 72$

$01 / 81$

$01 / 68$

$04 / 72$

$11 / 92$

$06 / 82$
E-1826-99CT

E-1867-99CT

E-1651-99CT

E-1379-45CT

E-1373-99CT

E-1368-99CT

E-1881-810CT

E-1387-99CT

\footnotetext{
* Black and white only
} 


\begin{tabular}{llll}
\hline Area & Image & Image & Identification \\
Name & Source & Date & Number \\
\hline
\end{tabular}

\section{Special Topics-Space, continued}

Solar System - mosaic of six planets

Apollo

$12 / 79$

E-996-99CT

Whole Earth - Saudi Arabia, Africa, S. Pole

Apollo 17

$04 / 72$

E-1357-99CT

Whole Earth - N./Central America - 50\% clouds

Apollo 17

N/A

E-1358-99CT

\section{Miscellaneous}

\begin{tabular}{|c|c|c|c|}
\hline Alaska/Canada - Mts. St. Elias/Logan, Yukon Territory & Landsat TM; DEM & $09 / 86$ & E-1897-99CT \\
\hline Augustine Volcano, AK & Landsat TM & $04 / 86$ & E-1708-44CT \\
\hline Black Hills, SD - mosaic & Landsat MSS & $09 / 79$ & E-1183-99CT \\
\hline Brazil - changes in forest inventory; three images & Landsat MSS & $1975,1986,1992$ & E-1926-99CT \\
\hline Cape Cod, MA & Space Shuttle Columbia & $04 / 81$ & E-1337-99CT \\
\hline Cape Cod, MA & Landsat MSS & $04 / 74$ & E-1827-99CT \\
\hline Chesapeake Bay, VA - image w/1:500,000-scale map & Landsat MSS & $10 / 72$ & E-1904-87CT \\
\hline China (Tsaidam Basin) - mosaic & Landsat MSS & $10 / 72$ & E-1056-810CT \\
\hline Global 1-km NDVI Greenness & AVHRR & $06 / 92$ & E-1912-99CT \\
\hline Global 1-km composite & AVHRR & $06 / 92$ & E-1913-99CT \\
\hline Grand Canyon, AZ & Landsat MSS & $10 / 73$ & E-1830-99CT \\
\hline Great Lakes Area & AVHRR & $09 / 87$ & E-1727-99CT \\
\hline Great Salt Lake, UT & Landsat TM & $11 / 84$ & E-1483-99CT \\
\hline Hubbard Glacier, AK & Landsat TM & $09 / 86$ & E-1566-99CT \\
\hline Hurricane Andrew - over Florida coast & AVHRR & $08 / 92$ & E-1891-99CT \\
\hline Hurricane Emily - over North Carolina coast & AVHRR & $08 / 93$ & E-1919-99CT \\
\hline Hurricane Felix - four scenes over east coast & AVHRR & $08 / 95$ & E-1940-99CT \\
\hline Hurricane Hugo - Eastern United States* & AVHRR & $09 / 89$ & E-751-8511BN \\
\hline Kuwait Oil Fires & Landsat TM & 05/91 & E-1862-99CT \\
\hline Lake Turkana, Ethiopia - monitoring change & Landsat MSS & $1973,1979,1989$ & E-1939-810CT \\
\hline Middle East - Persian Gulf/Red Sea & AVHRR & $08 / 90$ & E-1823-99CT \\
\hline Mississippi River Basin - flood scene & AVHRR & $07 / 93$ & E-1917-99CT \\
\hline Mississippi River Delta & Landsat MSS & $04 / 76$ & E-1831-99CT \\
\hline Mt. McKinley, AK & Aircraft close-up & $01 / 78$ & E-988-99CT \\
\hline Mt. McKinley, AK - digital mosaic & Landsat TM & $01 / 84$ & E-1481-99CT \\
\hline Mt. Pinatubo (volcano), Philippines & AVHRR & $07 / 91$ & E-1860-99CT \\
\hline Mt. Redoubt (volcano), AK & Landsat TM/DEM & $01 / 91$ & E-1817-99CT \\
\hline Mt. Saint Helens, WA* & Aircraft & $05 / 80$ & E-750-810BN \\
\hline New York State - Finger Lakes below Ontario & Landsat MSS & $04 / 76$ & E-1877-99CT \\
\hline Niagara Falls, NY - low oblique & EPA Aircraft & $08 / 74$ & E-1815-99CT \\
\hline North American DEM Data & DEM & $09 / 95$ & E-1993-99CT \\
\hline North American NDVI Vegetation Index & AVHRR & $03-11 / 90$ & E-1885-99CT \\
\hline North Pole 1-km NDVI (polar aspect) & AVHRR & $06 / 92$ & E-1923-99CT \\
\hline Persian Gulf - Kuwait Oil Fires & Shuttle & $04 / 91$ & E-1863-810CT \\
\hline Persian Gulf - Iraq/Saudi Arabia & AVHRR & $08 / 90$ & E-1822-99CT \\
\hline Red Sea & AVHRR & $11 / 88$ & E-1791-99CT \\
\hline Rocky Mountains, CO* & AVHRR & $07 / 87$ & E-757-99BN \\
\hline San Francisco Bay Area - mosaic & Landsat TM & $07-08 / 85$ & E-1814-99CT \\
\hline Somalia, Africa & AVHRR & $12 / 90$ & E-1900-99CT \\
\hline South America - mosaic & AVHRR & 1987-1989 & E-1819-99CT \\
\hline United States - mosaic & AVHRR & 1984-1986 & E-1649-99CT \\
\hline USA night scene with State boundaries & AVHRR mosaic & $09 / 87$ & E-1824-99CT \\
\hline Yellowstone Park, WY - fire boundaries & Landsat TM & $09 / 88$ & E-1772-99CT \\
\hline
\end{tabular}

* Black and white only

This is a list of satellite and high-altitude images of States, landforms, natural phenomena, and regions of the world. These photographs and images, often used by writers, editors, and broadcasters, provide spectacular views suitable for framing and display.

\section{Information}

For ordering information, contact any Earth Science Information Office (ESIC), or call 1-888-ASK-USGS.
For information on other USGS products and services, call 1-888-ASK-USGS, or visit the general interest publications Web site on mapping, geography, and related topics at erg.usgs.gov/isb/pubs/pubslists/.

For additional information, visit the ask.usgs.gov Web site or the USGS home page at www.usgs.gov.
For additional information, please contact:

Customer Services

EROS Data Center

Sioux Falls, SD 57198-0001

Phone: 605-594-6151

Fax: 605-594-6589

E-mail: custserv@edcmail.cr.usgs.gov

Some of these images are browsable at the EROS Selected Image Gallery Web site: http://edcwww.cr.usgs.gov/bin/ html_web_store.cgi 